\title{
A practical outcome scale for paediatric head injury
}

\author{
M Crouchman, L Rossiter, T Colaco, R Forsyth
}

\begin{abstract}
Traumatic brain injury (TBI) is the commonest cause of acquired disability in childhood. A major obstacle to the evaluation of acute and rehabilitative therapies after TBI is the lack of simple descriptors of outcome. We developed the King's Outcome Scale for Childhood Head Injury (KOSCHI), as a specific paediatric adaptation of the original adult Glasgow Outcome Scale (GOS). The KOSCHI expands the five category GOS to provide increased sensitivity at the milder end of the disability range. The GOS category of "persistent vegetative state" was replaced by "vegetative". "Good recovery" was allocated two categories, in acknowledgement of the long term importance of relatively minor sequelae in a developing child. The scale was quick and easy to use. Inter-rater reliability studies show that even with such an apparently simple scale, some training may be required. The KOSCHI provides a practical scale for paediatric head injury which will enable clinicians to describe rate and extent of recovery, and evaluate the effects of service and research interventions. (Arch Dis Child 2001;84:120-124)
\end{abstract}

Keywords: head injury; traumatic brain injury outcome scale

When Jennett and Bond published the Glasgow Outcome Scale (GOS) in $1975,{ }^{1}$ they made particular mention of the special developmental considerations in the assessment of outcome of traumatic brain injury (TBI) in children. Although the GOS has since become a standard outcome descriptor in adult TBI there remains no equivalent scale for use in children.

Head injury accounts for $5 \%$ of all paediatric

Neurosciences, King's College Hospital, Denmark Hill, London SE5 9RS, UK

M Crouchman

L Rossiter

T Colaco

Department of Child Health, Royal Victoria Infirmary,

Newcastle-upon-Tyne NE1 4LP, UK

R Forsyth

Correspondence to:

Dr Crouchman

marion.crouchman@kcl.ac.uk

Accepted 12 October 2000 the absence of physical deficits. ${ }^{3}$

A particular problem in the field of paediatric TBI research is the difficulty in developing simple yet meaningful descriptors of outcome. There are several challenges, particularly in describing outcome at the "higher" levels of functional independence or participation in society (see Discussion). We set out to produce a modification of the GOS which would
Table 1 TBI follow up checklist

- Neurological examination

- Epilepsy/medication

- Vision/hearing/smell

- Other medical (orthopaedic/maxillary-facial)

- Return to school

- Special educational needs status/input

- Mobility

- Behaviour (aggression, temper, disinhibition, hyperactivity)

- Mood (low, labile)

- Personality changes (drive, sense of humour)

- Social reintegration (family, peers)

- Concentration

- Short term memory

- Speech, language, word finding

- Sleep pattern (tiredness, nightmares, sleepwalking, fears, enuresis)

- Self help skills

- Appetite/weight

- Headaches/dizziness/tinnitus

- Evidence of post-traumatic stress in child, parents, other children

- Respite/family support

- Parental job change

- Rehousing needs

- Scarring/skull deformity

- Change in handedness

- Brain scan/EEG changes

- Rate of improvement-rapid, slowed, or static

provide a robust, simple description of outcome after paediatric TBI in the short, medium, or long term.

\section{Methods}

Information about the nature of head injury sequelae at various stages of recovery was obtained during the routine inpatient management and outpatient follow up of approximately 200 head injured children admitted to the paediatric wards of King's College Hospital and the paediatric neurosurgical unit over the period 1990-1997. More than 90\% had severe injury with abnormal intracerebral radiological findings on computed tomography. A simple checklist (table 1) was developed as an "aide memoire" for outpatient follow up. An hour long semistructured interview was held with the parents of six of the more severely injured children (aged 6-14 years) being followed three to five years after TBI to assess the extent and nature of problems encountered in the longer term. On the basis of this experience we modified the five categories of the GOS to produce the King's Outcome Scale for Childhood Head Injury (KOSCHI) (table 2).

\section{NOTES ON PARTICULAR CATEGORIES} head injury and includes brain stem death. ${ }^{4}$ The adjective "persistent", with its connotations of duration to date and implications of future persistence has been abandoned in connection with the vegetative state (category 2 ).
Death has to be directly attributable to the 
Table 2 KOSCHI category definitions

\begin{tabular}{|c|c|c|}
\hline Category & & Definition \\
\hline 1 & Death & \\
\hline 2 & Vegetative & $\begin{array}{l}\text { The child is breathing spontaneously and may have sleep/wake cycles. He may have non-purposeful or reflex movements of limbs } \\
\text { or eyes. There is no evidence of ability to communicate verbally or non-verbally or to respond to commands. }\end{array}$ \\
\hline 3 & Severe disability & $\begin{array}{l}\text { (a) The child is at least intermittently able to move part of the body/eyes to command or make purposeful spontaneous } \\
\text { movements; for example, confused child pulling at nasogastric tube, lashing out at carers, rolling over in bed. May be fully } \\
\text { conscious and able to communicate but not yet able to carry out any self care activities such as feeding. } \\
\text { (b) Implies a continuing high level of dependency, but the child can assist in daily activities; for example, can feed self or walk } \\
\text { with assistance or help to place items of clothing. Such a child is fully conscious but may still have a degree of post-traumatic } \\
\text { amnesia. }\end{array}$ \\
\hline 4 & Moderate disability & $\begin{array}{l}\text { (a) The child is mostly independent but needs a degree of supervision/actual help for physical or behavioural problems. Such a } \\
\text { child has overt problems; for example, } 12 \text { year old with moderate hemiplegia and dyspraxia insecure on stairs or needing help with } \\
\text { dressing. } \\
\text { (b) The child is age appropriately independent but has residual problems with learning/behaviour or neurological sequelae } \\
\text { affecting function. He probably should have special needs assistance but his special needs may not have been recognised/met. } \\
\text { Children with symptoms of post-traumatic stress are likely to fall into this category. }\end{array}$ \\
\hline 5 & Good recovery & $\begin{array}{l}\text { (a) This should only be assigned if the head injury has resulted in a new condition which does not interfere with the child's well } \\
\text { being and/or functioning; for example: } \\
\text { - Minor headaches not interfering with social or school functioning } \\
\text { Abnormalities on brain scan without any detectable new problem } \\
\text { Prophylactic anticonvulsants in the absence of clinical seizures } \\
\text { - Unsightly scarring of face/head likely to need cosmetic surgery at some stage } \\
\text { Mild neurological asymmetry but no evidence of affect on function of limb. Includes isolated change in hand dominance in } \\
\text { young child. } \\
\text { (b) Implies that the information available is that the child has made a complete recovery with no detectable sequelae from the } \\
\text { head injury. }\end{array}$ \\
\hline
\end{tabular}

All children pass through the vegetative stage, albeit often briefly, as they recover consciousness.

The original GOS category of severe disability has been divided into two. A child in category 3 a will show some evidence of awareness (for example, visual tracking) although responsiveness (for example, to command) may be unreliable. In category $3 \mathrm{~b}$ the child has some limited functional independence but requires adult help or supervision for most activities. He may have simple, yet meaningful, possibly non-verbal communication. As with the GOS, severe cognitive damage may place a child in one of these categories despite good motor recovery.

The GOS defines "good recovery" (categories $5 \mathrm{a}, 5 \mathrm{~b}$ ) in terms of the resumption of previous life (although not necessarily full return to work), notwithstanding minor neurological or psychological deficits. However, the authors acknowledge that head injury in childhood may produce deficits which increase with continuing development. Minor residual deficits are potentially much more destructive to children, and our scale acknowledges the importance of this, particularly in relation to social and learning behaviour. We therefore have stricter crite-

Table 3 Components of the functional scoring system

\begin{tabular}{lll}
\hline Mobility & 3 & Normal \\
& 2 & Impaired \\
Communication & 1 & Fully dependent \\
& 4 & Normal \\
& 3 & Impaired \\
& 2 & Very limited (e.g. "yes/no") communication \\
Mood, behaviour, personality & 1 & Absent \\
Disinhibition & 2 & Normal \\
Danger awareness & 1 & Problems arising \\
Self care & 2 & Absent \\
& 1 & Present \\
Cognition, memory, and concentration & 2 & Aware \\
& 1 & Unaware \\
& 3 & Age appropriate independence \\
& 2 & Needs assistance, task modification, or prompts \\
& 1 & Fully dependent \\
& 2 & Minor problems \\
& 1 & Marked problems \\
\hline
\end{tabular}

ria for this category and have divided the scale into two subcategories (see table 2).

\section{INTER-RATER RELIABILITY}

An extended inter-rater reliability exercise was performed. Six observers with professional interests in childhood head injury (two paediatric neurologists, two clinical nurse specialists, a health economist, and a medical social worker, all with specialist experience in the field) independently assigned KOSCHI categories (from 2 to $5 \mathrm{~b}$ ) to 90 child survivors of head injury. Ratings were made from the information in anonymised routine outpatient follow up clinic letters. Inter-rater reliability was calculated using a modified kappa statistic. The kappa statistic measures the agreement between observers rating samples on a nominal scale. The original Cohen statistic ${ }^{5}$ considered the case of two observers. Fleiss ${ }^{6}$ and Dunn ${ }^{7}$ described analogous statistics for the case of multiple observers; these were used in this study.

Further studies of inter-rater differences required treatment of the KOSCHI category score as a continous scale (see Discussion). See below for justification for this transformation. KOSCHI scores ( 1 to $5 \mathrm{~b}$ ) were mapped to a continuous scale (1 to 8). Observers' performances were compared by calculating the residual between each observer's rating of each child and the mean of all observers' ratings for that child.

CONSTRUCT VALIDITY

In order to identify the factors determining KOSCHI scale assignments, a simple functional score based on likely candidates was devised (table 3). Using a separate dataset of 80 outpatient clinic letters, children's functional scores (from table 3) and overall KOSCHI category were independently assigned by two observers. Functional scores were related to overall KOSCHI category by multinomial logit and conventional regression. 
Table 4 Observer effects

\begin{tabular}{lll}
\hline Observer & Mean residual (SEM) & p value \\
\hline 1 & $0.011(0.038)$ & NS \\
2 & $-0.522(0.076)$ & $<0.0001$ \\
3 & $-0.011(0.045)$ & NS \\
4 & $-0.022(0.040)$ & NS \\
5 & $-0.011(0.052)$ & NS \\
6 & $0.033(0.042)$ & NS
\end{tabular}

Mean residuals for each observer are relative to the "gold standard" KOSCHI score (calculated as the mean observers' scores excluding observer 2). KOSCHI scores have been treated as a continuous variable ( 1 to 8 ) for this analysis. Statistical significance of difference from zero by $t$ test.

Table 5 Inter-rater reliability

\begin{tabular}{lll}
\hline KOSCHI category & Description & Fleiss kappa statistic \\
\hline 1 & Dead & N/A \\
2 & Vegetative & 0.88 \\
3a & Severe disability & 0.63 \\
3b & & 0.33 \\
$4 \mathrm{a}$ & Moderate disability & 0.28 \\
$4 \mathrm{~b}$ & & 0.51 \\
$5 \mathrm{a}$ & Good recovery & 0.53 \\
$5 \mathrm{~b}$ & Intact recovery & 0.66 \\
\hline
\end{tabular}

Kappa statistics by KOSCHI category. Inter-rater reliability exercises were performed only on survivors, hence there are no KOSCHI category 1 ("dead") observations. Values calculated after omitting observer 2 show a qualitatively very similar pattern.

\section{Results}

INTER-RATER RELIABILITY

Treating KOSCHI categories as a continuous variable showed a systematic tendency of observer 2 to rate children as more severely disabled; mean of residual scores for observer 2 was -0.52 (SEM 0.08) KOSCHI grades $(\mathrm{p}<0.0001 ; t$ test). This tendency was replicated in a duplicate analysis of 99 children (data not shown). No other systematic effects were noted (table 4).

Treating the KOSCHI categories as a categorical variable, the kappa score for six observers' ratings of 90 children was 0.51 . Excluding observer 2, the kappa statistic among the remaining five observers was 0.58 . Table 5 shows kappa statistics for each grade. Observer agreement was highest for very severely disabled children. Agreement was poorest in categories $3 \mathrm{~b}$ and $4 \mathrm{a}$.

\section{CONSTRUCT VALIDITY}

Regression studies of independently assigned functional scores (table 3) and overall $\mathrm{KO}-$ SCHI category confirmed that it is reasonable to treat KOSCHI categories (1 to $5 b$ ) as an eight point interval scale, should this be required. In the dataset of 80 patients used for this analysis, only one was in KOSCHI category 2 , and only two in category $3 \mathrm{~b}$. These patients were almost completely identified by their mobility, communication, and self care scores. A regression involving the remaining 77 patients showed that the KOSCHI category could be predicted by a simple linear function of the component scores with increased weighting given to the self care score. While the total "functional score" calculated in this way was highly correlated with KOSCHI grade $(\mathrm{R}=0.82)$ the residual standard deviation was considerable at 0.7 of a KOSCHI grade.

\section{Discussion}

In the forthcoming revision (ICIDH2) of the WHO Classification of Impairments, Disabilities, and Handicaps, the concepts of activity (functional at the level of the individual, in a standardised, optimal environment) and participation (determined by the interaction between the individual's potential and real world environmental constraints) replace those previously denoted by the terms "disability" and "handicap". There are several particular challenges in describing outcome after childhood TBI at these higher levels. Measures must relate to age appropriate expectations, and since the developmental process continues (albeit modified) after the injury, the shortfall between current functional status and age dependent "normality" is dynamic. There is also a dynamic relation between the component parts of the total morbidity seen after TBI. Physical, behavioural, and cognitive components make varying contributions to the overall morbidity, depending on age at injury, ${ }^{8}$ interval since injury, injury severity, ${ }^{9}$ and injury independent factors. ${ }^{111}$

The GOS is completed on the basis of the clinician's assessment of the best available clinical information. We wished to evaluate the KOSCHI in a "real world" setting where scores were being made retrospectively on the basis of information in routine clinic letters. The limited detail with which children's abilities were discussed in these letters was, we feel, responsible in large part for the modest inter-rater reliability. In a research setting, in which KOSCHI gradings are being made on the basis of systematically collected information, inter-rater reliability is likely to be considerably higher.

In general terms, the kappa statistic quantifies the observed degree of interobserver agreement and compares the excess of observed agreement over that expected by chance to the maximum possible excess of observed agreement over chance-expectation. A major disadvantage of the kappa statistic is therefore its dependence on the chance expectation of interobserver agreement, which is itself dependent on the number of observers, observations, and possible categories. Thus conventional criteria of "substantial" (kappa 0.60.79) or "almost perfect" agreement (kappa $>0.8$ ) relating to two observers are inapplicable in this context. Interpretation of the "adequacy" of the kappa of $0.51-0.58$ seen in this study is therefore difficult.

An additional disadvantage of the kappa statistic is that no account is taken of the magnitude of interobserver disagreement. Discrepancy of any magnitude (even one grade step) represents "disagreement". To overcome this difficulty we performed the continuous variable analyses (table 4 ). These show the potential for significant systematic errors in rating, even with such an apparently simple instrument as the KOSCHI. Before the KOSCHI is used in clinical or research practice, we would recommend a team of potential observers to perform pilot comparisons of their 
rating practices to ensure that they are scoring consistently.

Conceptually, the suggestion that "recovery after head injury" can be captured in a unidimensional scale could be challenged. The difference between KOSCHI grades 1 (dead) and 2 (vegetative) is one of physiological function; between 2 and 3a, a matter of awareness of and response to external stimuli; and between grades $3 \mathrm{~b}$ to $5 \mathrm{a}$ essentially degree of functional independence. Nevertheless the multinomial logit regression lends support to use of a unidimensional scale; an approach related to the more commonly used analyses based on Rasch item response theory. The construct validity studies are reassuring. The slightly greater weight given to the self care component is explicable in terms of the criteria defining the grades $3 \mathrm{~b}$ to $5 \mathrm{a}$, which are largely based on functional independence.

The authors of the GOS recognised the difficulties in applying it to children. We set out to produce a modification of the GOS which would provide a robust, simple scale for short, medium, and long term paediatric head injury outcome. Our requirements included compatibility with the GOS to allow comparison with adults, and facilitate the transition of follow up into adulthood; applicability at all ages; and simplicity of use. It can be completed by direct observation or from routine medical records, prospectively or retrospectively. Sensitivity to apparently minor sequelae of $\mathrm{TBI}$, which in children may have long term impacts, was a major concern. With experience, children can be allocated to the appropriate KOSCHI category within a matter of minutes.

The scale places a high emphasis on concentration, behaviour, and disinhibition, which are common problems in this group of children. Disinhibition was described by Rutter ${ }^{12}$ as the behavioural outcome most typical of acquired brain injury. It is highly socially disabling, and results in continuing dependency even in the absence of physical sequelae. Injuries to the frontal lobes of the brain are common after TBI, although they may not manifest until puberty. ${ }^{13}$ There is increasing awareness of the long term hidden effects of this type of damage to immature brains. ${ }^{14-16}$ For example, a child who has been allowed to return to mainstream school and apparently "fully recovered" (category $5 \mathrm{a}$ or $5 \mathrm{~b}$ ) may start to fail as educational expectations and demands increase, the impact of the injury on new learning ${ }^{17} 18$ becomes apparent, and he slips back into category $4 \mathrm{~b}$. The development of secondary behaviour problems may contribute to this decline in school achievement. ${ }^{19}$

There is inevitably some potential for ambiguity in such a simple instrument. For example, a child with minor head injury who has a partial spinal cord transection will acquire a functional disability which could place him in category $4 \mathrm{a}$, irrespective of complete recovery from the brain injury per se. Where possible, information on the sequelae of the child's brain injury rather than of other injuries, should be used. A much more common problem encountered is the child with premorbid learning and/or behaviour problems and as a general rule, assignment to a category should be based on change in function following TBI. Such ambiguities will be less relevant to summary outcome data for large groups.

Although the scale was originally designed for children aged 2 to 16 years of age, there is no reason why it should not be used under the age of 2 years with the caveat that problems in this young age group are commonly underestimated. Falls and intentional shaking injuries are important causes of TBI in this group. Even if the child scores normally at this young age it must be borne in mind that problems such as dyspraxia or language disorders may emerge with time, and the child may regress through the categories as the path of development becomes more aberrant. This is particularly true of infants with subdural haemorrhages, ${ }^{20}$ in whom shaking causes widespread axonal shearing. ${ }^{21}$

The KOSCHI is a short hand description of the child's level of functioning at a point in time. As such it provides a useful clinical tool for documenting recovery in the individual child, as well as for monitoring the burden of disability caused by TBI, for service planning, and for evaluating rehabilitation programmes and the effects of service and research intervention. We hope that, like the GOS, it will be further validated by comparison with outcome predictors such as the Glasgow Coma Score and measurements of posttraumatic amnesia.

Thelma Colaco was supported by a grant from the Joint Research Committee. We thank S Beljung for preparation of the typescript, C Hawley, $\mathrm{H}$ Greenberg, E Jones and C Pickthall for their participation in the inter-rater reliability exercises, and $\mathrm{M}$ Aitken for statistical advice.

1 Jennett $\mathrm{B}$, Bond $\mathrm{M}$. Assessment of outcome after severe brain damage. A practical scale. Lancet 1975;1:480-4.

2 Sharples PM. Head injury in children. In: Little and Ward Platt, eds. Injury in the young. Cambridge: Cambridge University Press, 1998:151-75.

3 Crouchman MR. Recovery, rehabilitation and the neuropsychological sequelae of head injury. In: Little and Ward Platt, eds. Injury in the young. Cambridge: Cambridge University Press, 1998:263-99.

4 British Paediatric Association. Diagnosis of brain stem death in infants and children. Working party report. London: British Paediatric Association, 1991 .

5 Cohen JA. A coeffficient of agreement for nominal scales. Educational and Psychological Measurement 1960;20:37-46.

Fleiss JL. Measuring nominal scale agreement among many raters. Psychol Bull 1971;76:378-82.

7 Dunn G. Design and analysis of reliability studies. London: Arnold, 1989:154.

8 Anderson V, Moore C. Age at injury as a predictor of outcome following pediatric head injury: a longitudinal perspective. Child Neuropsychol 1995;1:187-202.

9 Fletcher JM, Ewing-Cobbs L, Francis DJ, Levin HS. Variability in outcomes after traumatic brain injury in children: a developmental perspective. In: Broman SH, Michel ME, eds. Traumatic head injury in children. New York: Oxford University Press, 1995:22-39.

10 Rivara JB, Jaffe KM, Polissar NL, et al. Family functioning and children's academic performance and behavior probems in the year following traumatic brain injury. Arch Phys Med Rehabil 1994;75:369-79.

11 Rivara JB, Jaffe KM, Polissar NL, et al. Predictors of family functioning and change 3 years after traumatic brain injury in children. Arch Phys Med Rehabil 1996;77:754-64.

12 Rutter M. Psychological sequelae of brain damage in children. Am f Psychiatry 1981;138:1533-44.

13 Esliger PJ, Grattan LM, Damazio H, Damasio AR. Developmental consequences of childhood frontal lobe damage. Arch Neurol 1992;49:764-9.

14 Brown G, Chadwick O, Shaffer D, et al. A prospective study Brown G, Chadwick O, Shaffer D, et al. A prospective study
of children with head injuries. III. Psychiatric sequelae. Psychol Med 1981;11:63-78.

15 Dennis M, Barnes MA. Knowing the meaning, getting the point, bridging the gap, and carrying the message. Aspects 
of discourse following closed head injury in childhood and adolescence. Brain Lang 1990;39:428-46.

16 Levin HS, Kraus MF. The frontal lobes and traumatic brain injury. Fournal of Neurology, Psychology, Clinical Neurological Sciences 1994;6:443-54.

17 Gulbrandsen GB. Neuropsychological sequelae of light head injuries in old children six months after trauma. $f$ Clin Neuropsychol 1994;6:257-68.

18 Ewing R, McCarthy D, Gronwall D, et al. Persistent effects of minor head injury observable during hypoxic stress. $\mathcal{F}$ Clin Neuropsychol 1980;2:147-55.
19 Brink JD, Garrett AL, Hale WR, et al. Recovery of motor and intellectual functioning in children sustaining severe head injuries. Dev Med Child Neurol 1970;12:56571

20 Bonnier C, Nassogue MC, Evrard P. Outcome and prognosis of whiplash shaken infant syndrome; late consequences after a symptom-free interval. Dev Med Child Neurol 1995; 37:943-56.

21 Duhaime AC, Gennarelli TA, Thibauld LE, et al. The shaken baby syndrome, a clinical, pathological and biomechanical study. F Neurosurg 1987;66:409-15.

\section{EUROPE CALLING}

\section{Eradication of poliomyelitis: where do we stand in Europe?}

In 1988, the World Health Assembly adopted the goal of global eradication of poliomyelitis by immunisation, a target which was to originally be achieved by the end of the year 2000. Eradication of polio is now also one of three top priorities of the World Health Organisation, along with reduction of smoking and control of malaria. Since 1988, substantial progress has been made. Mass immunisation campaigns and national immunisation days have been performed repeatedly in many countries with remarkable success - for example, the last case of polio in the Americas occurred in 1991 and the last case occurred in the Western Pacific region in 1997.

In the meantime, the date for eradication of the disease has been moved to the end of 2002 and intensified activities are needed if this goal is to be achieved in the remaining endemic countries of Africa ( $>2800$ cases in 1999) and Asia (>3300 cases in 1999).

In the WHO European region, which comprises 51 countries in Europe and the former Soviet Union, no cases of polio have been reported since November 1998. This is very promising and has led to a change in immunisation policy in many European countries: where live attenuated oral vaccine (OPV) has been used predominantly, vaccine associated paralytic poliomyelitis-though very rareoccurs more frequently than wild type virus infection. Consequently, immunisation with OPV has been widely replaced by injectable inactivated polio vaccine.

However, given that 99 asymptomatic infections stand behind each notified "case" (acute flaccid paralysis caused by one of the three poliomyelitis viruses), great care is needed before a region can be declared free of poliomyelitis. Sensitive surveillance programmes need to be in place to ascertain that all cases of acute flaccid paralysis are reported and that a diagnosis of poliomyelitis is ruled out by appropriate tests. This is the basis for certifying that a region is free of polio, the goal expressed by the WHO for the European region by the year 2001 .

What remains to be done? The gold standard is to monitor all cases of non-traumatic acute flaccid paralysis in children and adolescents under 15 years of age. Two stool samples, separated by 24-48 hours and collected within 14 days of onset, need to be shown to be free of poliomyelitis virus. A country's surveillance system is considered sensitive enough if at least one case of acute flaccid paralysis per 100000 children under 15 years of age is detected each year. For example, Finland has accomplished this with exactly one report in 1998, while Germany (with 0.47 cases), like many other European countries, is still struggling although the reported rates are gradually increasing.

The ultimate goal is to stop immunisation after poliomyelitis has been eliminated, as was done after the eradication of smallpox. If all countries are certified free of polio by 2005, WHO hopes to stop immunisation by 2010 . Is this realistic? Probably not, as too many open questions remain. The most difficult issue is the likelihood that many countries will keep stocks of poliomyelitis virus strains that could be used as weapons in a biological warfare. By definition, this is not compatible with the term "eradication". It is very possible that immunisation against poliomyelitis will stay with us for a long period of time.

ULRICH HEININGER European Associate Editor 